\title{
Expression Patterns of Adhesion Molecules in Human Gingival Epithelium
}

\author{
Setsuko Hatakeyama, Yuko Oikawa, Takashi Yaegashi, Hideaki Fujiwara, Izuru Furuya, and Masanobu Satoh \\ ${ }^{1}$ Department of Oral Pathology, ${ }^{2}$ Department of Periodontology, School of Dentistry, Iwate Medical University, 19-1 Uchimaru, Morioka, \\ 020-8505 Japan
}

\begin{abstract}
The gingival epithelium is the physiologically important interface between the bacterially colonized gingival sulcus and periodontal soft and mineralized connective tissues requiring protection from exposure to bacteria and their products. However, the molecules comprising the gingival epithelial cell junction remain poorly characterized. Thus, the aim of the present study was to characterize the desmosome-associating proteins (desmoglein 1 and 3), desmosome-associating cytoskeleton (keratins), and tight junction-associating protein (claudin-1) within the oral gingival epithelium (OGE), sulcular epithelium (SE), and junctional epithelium (JE). Gingival epithelia excised at therapeutic flap surgery from patients with periodontitis were used to examine expression of adhesion molecules by immunofluorescence. In the OGE and SE, but not JE, desmoglein 1 was more abundant in the cell-cell contact sites of the upper than the suprabasal layer, while desmoglein 3 and desmoplakin were present in the cell-cell contact sites in all layers of the JE as well as the OGE and SE. Keratin 14 and 19, but not keratin 13 and 4, were present in the JE. Claudin-1 was expressed only in the intermediate layers in the uppermost flat layers in the OGE. These data indicated that the JE contained only few desmosomes composed of desmoglein 3. Thus, it is thought that the anchoring junction connecting JE cells is not firm, causing widened intercellular spaces in the JE. In contrast, the OGE, which has tight junctions, functions as a barrier.
\end{abstract}

Key words: desmoglein, junctional epithelium, claudin-1, Immunohistochemistry

\section{Introduction}

The epithelium adjacent to the tooth can be classified into three anatomical types: the oral gingival epithelium (OGE), the sulcular epithelium (SE) and the junctional epithelium (JE). Each type of epithelium is structurally and functionally unique. Of them, the JE contains only several layers of cells that attach to the tooth surface (1) and has no keratinizing epithelial cell layer at the free surface and wide intercellular spaces and may thereby provide a pathway for fluid and transmigrating leukocytes from the gingival connective tissue to the gingival sulcus (2). Thus, characterization of the adhesion structures of the JE that support its multilayered architecture and that create wide intercellular spaces is an important step in understanding the normal functioning of the JE.

Cell junctions are assembled at points of cell-cell and cell-matrix contact in all tissues and are cell-cell junctions are abundant in squamous epithelia. In general, of cell-cell junctions, desmosomes act as anchoring adhesion structures in various tissues including stratified epithelium (3). Thus, in the present study, immunohistochemical localization of the cell adhesion molecules constituting desmosomes within the OGE, SE, and JE was examined. Furthermore, claudin-1 (major tight junctionassociating proteins (4)) was also studied.

Tissue samples

\section{Materials and methods}

Samples of gingival tissues were obtained from adult patients with periodontitis undergoing flap operation at the Dental Hospital of Iwate Medical University. Written informed consent was obtained from all patients prior to operation, and the ethical committee of Iwate Medical University approved all protocols.

Correspondence to Setsuko Hatakeyama Ph.D., Department of Oral Pathology, School of Dentistry, Iwate Medical University, 19-1 Uchimaru, Morioka 020-8505, Japan. Tel: +81-19-651-5111, Fax: +81-19-621-3321, e-mail: hsetsuko@iwate-med.ac.jp
Excised samples were immediately cut along the vertical axis of the tooth and embedded in O.C.T. Compound (Tissue-Tek, Sakura Fine technical Co. Ltd, Tokyo, Japan) before being snap-frozen in acetone solution cooled with dry ice, and $5 \mathrm{~mm}$ sections were cut.

\section{Immunofluorescence}

We examined the following molecules: 1) desmosomeassociating proteins: desmoglein 1, desmoglein 3, and desmoplakin, 2) the intracellular cytoskeleton, keratins, and 3) tight junction-associating protein (Table 1). Sections were fixed in ethanol before immunoreaction with primary antibodies for 10 min at $4 \mathrm{C}$. Incubation with primary antibodies was performed for two hours at room temperature. Then, after washing in phosphate-buffered saline with Tween 20 (PBST), sections were incubated with either fluorescein isothiocyanate (FITC)conjugated goat anti-mouse antibody (Kirrkegaard \& Petty Laboratories Inc., Gaithersburg, USA), Alexa Fluor $488^{\circledR}$ conjugated goat anti-rabbit IgG, or Alexa Fluor $594^{\circledR}$-labeled donkey anti-goat IgG (Molecular Probes Inc., Eugene, USA) for 1 hour at room temperature. Finally, specimens were mounted in mounting medium and then inspected with a laser scanning confocal microscope (LSM510, Zeiss, Göttingen, Germany).

Keratin 4, 13, 14, and 19

Results

Inspection of areas of healthy gingival epithelia revealed intact OGE, SE, and JE and a small number of chronic inflammatory cells in the sub-epithelial connective tissue. Different patterns of keratin expression were present in the three regions of the gingival epithelium (Table 2). In every specimen, the cytoplasm of all layers of OGE, SE, and JE cells exhibited strong staining with anti-keratin 14 antibodies. In contrast, only basal cells of the OGE and SE, and almost all cells in JE were clearly positive for keratin 19. 
International symposium of Maxillofacial \& Oral Regenerative Biology in Okayama 2005

Table 1. Antibodies employed in this study

\begin{tabular}{lllcl}
\hline \multicolumn{1}{c}{ Antibodies } & Source & Clone & Immunnized animals & Opiticaldilution \\
\hline Claudin-1 & Santa Cruz & & rabbit & $1: 50$ \\
Keratin 4 & PROGEN & 6B10 & mouse & $1: 50$ \\
Keratin 13 & Cappel & MIC7 & mouse & $1: 50$ \\
Keratin 14 & YLEM & IL002 & mouse & $1: 50$ \\
Keratin 19 & DAKO & RCK108 & mouse & $1: 50$ \\
Desmoglein 1 & PROGEN & DG3.10 & mouse & $1: 50$ \\
Desmoglein 3 & Santa Cruz & & rabbit & $1: 50$ \\
desmoplakin & PROGEN & & goat & $1: 50$ \\
\hline
\end{tabular}

Immunostaining for keratin 13 was found in prickle cells in the OGE and SE, but not in the JE. Finally, Immunostaining for keratin 4 was present in the marginal epithelium and the SE, but not in the OGE or JE.

The desmosome proteins: desmoglein 1, desmoglein 3, and

Immunostaining experiments suggested that desmoglein 1 was abundant in the cell-cell contact sites of the spinous and suprabasal layers of the OGE and SE but was not present in the JE. In contrast, desmoglein 3 was present in the cell-cell contact sites in all layers involving the basal layers of the OGE, SE, and also in the JE. The uppermost layers of the SE and OGE were negative for desmogleins. Furthermore, desmoglein 3 was not present in the cell membrane facing the basement membrane of the basal cells. Finally, desmoplakin was present in cell-cell contact sites in all layers of the OGE, SE, and JE.

The occluding junction proteins, claudin-1.

Claudin-1 was present only in the uppermost intermediate layers of the OGE.

\section{Discussion}

Stratified squamous epithelium is characterized by the presence of abundant desmosomes. Specific desmoglein isoforms are expressed at different stages of keratinocyte differentiation (5). Desmoglein 1, which is a target protein in the autoimmune disease pemphigus foliaceus (6), is restricted to the upper spinous layers of the epidermis. In contrast, desmoglein 3 , which is involved in the pathogenesis of pemphigus vulgaris (7), is mainly localized in the basal and spinous layers of the epidermis. Although it has been reported that the JE contains few desmosomes, the expression pattern of desmogleins in the JE has not previously been described. The present study is the first to demonstrate that desmoglein 3, but not desmoglein 1, was localized to the JE. This indicates that desmosomes in the JE are comprised of desmoglein 3 but not desmoglein 1 and is probably consistent with the small size and number of desmosomes present in the JE when compared with the OGE $(2,8)$. Incomplete desmosome structure in the JE leads to immaturity of keratinocyte differentiation and wide intercellular spaces. The keratin expression of stratified epithelia varies according to the stage of cellular differentiation (9). In the gingival epithelium, keratin 19 was localized to the basal cells of the OGE and SE and was present in all cells of the JE, while keratin 4 and keratin 13, known as mucous type keratins, were not expressed. These data indicate that the JE is composed of undifferentiated epithelial cells that correspond to basal cells in the keratinocyte differentiation lineage.

Tight junctions function to seal cells together, to maintain polarity of cells (3), and to separate the luminal space from the mesenchymal space. Constitutive tight junction proteins and tight junction-related structures have also been identified in squamous stratified epithelia, including the epidermis $(4,10)$, where they are predominantly present in the stratum granulosum. In this study, claudin-1 was present in the intermediate portion of a few of the uppermost epidermal layers of the oral mucosa. These data suggest that the tight junction is probably a typical structure in the uppermost layer that is equivalent to the stratum granulosum in stratified epithelium and functions as a barrier (11).

\section{Acknowledgements}

This study was supported in part by a Grant-in-Aid for Scientific

Table 2 Immunofluorescense of cell-adhesion molecules in human gingival epithelia

\begin{tabular}{lllll} 
& Molecules & JE & SE & OGE \\
\hline $\begin{array}{llll}\text { Occluding junctions } \\
\text { tight junction } \\
\text { desmosome }\end{array}$ & claudin-1 & $(-)$ & $(-)$ & $(+)$ uppermost layers \\
& keratin 19 & $(+)$ all layers & $(+)$ only basal layer & $(+)$ only basal layer \\
& keratin 14 & $(+)$ all layers & $(+)$ all layers & $(+)$ all layers \\
keratin 13 & $(-)$ & $(+)$ spinous layers & $(+)$ spinous layers \\
& kerain 4 & $(-)$ & $(+)$ all layers & $(-)$ \\
& desmoglein 1 & $(-)$ & $(+)$ spinous layers & $(+)$ spinous layers \\
& desmoglein 3 & $(+)$ all layers & $(+)$ all layers & $(+)$ all layers \\
\hline
\end{tabular}

OGE: oral gingival epithelium, SE: sulcular epithelium, JE: junctional epithelium

$(+)$ : positive, \pm$\} \cdot j$ : weakly positive, $(\cdot)$ : negative 
Research (C) No. 15591944, Open Research Project (2001-2005) from the Ministry of Education, Culture, Sports, Science, and Technology, Japan.

\section{References}

1. Hormia M, Owaribe K, and Virtanen I.: The dento-epithelial junction: cell adhesion by type I hemidesmosomes in the absence of a true basal lamina. J Periodontol, 72: 788-797, 2000

2. Bosshardt DD and Lang NP.: The junctional epithelium: from health to disease. J Dent Res, 84: 9-20, 2000

3. Alberts B, Johnson A, Lewis J, Raff M, Roberts K, and Walter P.: Molecular Biology of the Cell. $4^{\text {th }}$ edn. New York: Garland Science 1066-1125, 2002

4. Langbein L, U-F Page, C grand, C Kuhn, S Praetzel, I Moll, R Moll, and Franke WW.: Tight junction-related structures in the absence of a lumen: occludins, claudins and tight junction plaque proteins in densely packed cell formations of stratified epithelia and squamous cell carcinomas. Eur J Cell Biol, 82: 385-400, 2003

5. Christiano AM, and Uitto J.: Molecular complexity of the cutaneous basement membrane zone. Exp Dermatol, 5: 1-11. 1996
6. Mahoney MG, Wang Z, Rothenberger K, Koch PJ, Amagai M, and Stanley JR.: Explanations of the clinical and microscopic localization of lesions in pemphigus foliaceus and vulgaris. J Clin Invest, 104: 461-468, 1999

7. Amagai M, Koch PJ, Nishikawa T, and Stanley JR.: Pemphigus vulgaris antigen (desmoglein 3 ) is localized in the lower epidermis, the site of blister formation in patients. J Invest Dermatol, 106: 351-355, 1996

8. Shimono M, Ishikawa T, Enokiya Y, Muramatsu T, Matsuzaka K, Inoue T, Abiko Y, Yamada T, Kido MA, Tanaka T, and Hashimoto S.: Biological characteristics of the junctional epithelium. J Electron Microsc, 52: 627-639, 2003

9. Mackenzie IC, Rittman G, Gao Z, Leigh I, and Lane EB.: Patterns of cytokeratin expression in human gingival epithelia. J Periodont Res, 26: 468-478, 1991

10. Morita K, Tsukita S, and Miyachi Y. Tight junction-associated proteins (occludin, ZO-1, claudin-1, claudin-4) in squamous cell carcinoma and Bowen's disease. Br J Dermatol, 151: 328334, 2004

11. Furuse M, Hata M, Furuse K, Yoshida Y, Haratke A, sugitani Y, Noda T, Kubo A, and Tsukita S.: Claudin-based tight junctions are crucial for the mammalian epidermal barrier: a lesson from claudin-1-deficient mice. J Cell Boil, 156: 10991111, 2002 